University of Nebraska - Lincoln

DigitalCommons@University of Nebraska - Lincoln

Faculty Publications in Computer \& Electronics Electrical \& Computer Engineering, Department Engineering (to 2015)

2012

\title{
Impact Of Wireless Communication Delay On Load Sharing Among Distributed Generation Systems Through Smart Microgrids
}

\author{
Song $\mathrm{Ci}$ \\ University of Nebraska-Lincoln, sci2@unl.edu \\ Junjie Qian \\ University of Nebraska-Lincoln \\ Dalei Wu \\ University of Nebraska-Lincoln, dalei-wu@utc.edu \\ Ali Keyhani \\ Ohio State University
}

Follow this and additional works at: https://digitalcommons.unl.edu/computerelectronicfacpub

Part of the Computer Engineering Commons

Ci, Song; Qian, Junjie; Wu, Dalei; and Keyhani, Ali, "Impact Of Wireless Communication Delay On Load Sharing Among Distributed Generation Systems Through Smart Microgrids" (2012). Faculty Publications in Computer \& Electronics Engineering (to 2015). 74.

https://digitalcommons.unl.edu/computerelectronicfacpub/74

This Article is brought to you for free and open access by the Electrical \& Computer Engineering, Department of at DigitalCommons@University of Nebraska - Lincoln. It has been accepted for inclusion in Faculty Publications in Computer \& Electronics Engineering (to 2015) by an authorized administrator of DigitalCommons@University of Nebraska - Lincoln. 


\title{
ReCent AdVANCES IN \\ WireleSS TeChNOLOGIES fOR SMART GRID
}

\section{IMPACT OF WIRELESS COMMUNICATION DELAY ON LOAD SHARINg AMONg DISTRIBUTEd GENERATION SYSTEMS THROUGH SMART MICROGRIDS}

\author{
SONG CI AND JUNJIE QIAN, UNIVERSITY OF NEBRASKA-LINCOLN AND \\ CHINESE ACADEMY OF SCIENCES \\ DaleI Wu, MASSACHUSETTS INSTITUTE OF TECHNOLOGY \\ Ali KeyHani, OHIO State UnIVERsity
}

Output filter circuit

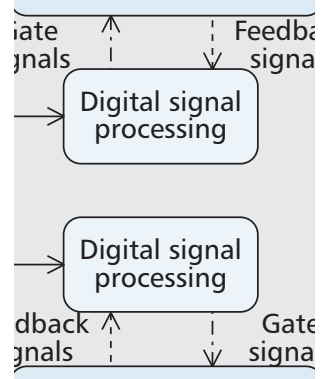

Output filter circuit

The authors study the performance of distributed power inverters under the scenario of wirelessenabled smart microgrids. They study the power inverter performance and propose an optimal controller.

\begin{abstract}
Load sharing among distributed generation systems (DGS) is one of the most important topics in wireless-enabled smart microgrids, where a set of power inverters at different geographical locations work coordinately and collaboratively through wireless networks to meet the load requirement. Parallel power inverters operated in distributed generation systems rely on the underlying wireless communication channels to exchange power information among them for load sharing control. However, existing work in the area of load sharing has largely ignored the effect of wireless communication delay. Study has shown that the time-varying wireless communication delay may have a significant impact on the performance of power inverters. In this work, we aim to fill this gap and study the performance of distributed power inverters under the scenario of wireless-enabled smart microgrids. We first study the power inverter performance by taking into account the time-varying wireless communication delay. Then, we propose an optimal controller to overcome the delay problem and to provide a smooth load sharing performance. Both theoretical and simulation results show the effectiveness and responsiveness of the proposed controller design.
\end{abstract}

\section{INTRODUCTION}

Due to the increasing concern on environment and the rising fuel price, smart microgrids have been receiving more and more research attentions recently. Conceptually, a smart microgrid is made up of various generation sources (fuel cells, energy storage devices, small wind turbines, solar/photovoltaic systems, small hydro turbines) [1], different energy consumption devices, and/or energy management platform. Subsystems in smart microgrids are interconnect- ed by local microgrids and wireless communication networks. Energy from both local and remote energy sources flows along the microgrids, while energy management information needed to control the entire system is transmitted through the wireless communications networks.

Introduction of such smart microgrids is very attractive for campuses, commercial facilities and residential communities, because they can significantly reduce energy cost and carbon emission by managing distributed generation systems to supply load and optimize energy use. Generally speaking, the technologies used in smart microgrids are based on two aspects: power electronics and wireless communications. All DGS require power converters, PWM techniques and electronic control units. Wireless communications is essential for exchange of information in smart microgrids, including the system operation information and the power control information necessary for power inverters control.

Since the most important feature of smart microgrids is to supply load with distributed sources, load sharing has been widely used in smart microgrids. A typical load sharing scenario in smart microgrids is shown in Fig. 1 where a wireless community mesh network has been used for control signal exchange. Different households with their own distributed generation systems (DGSs) share the energy generated across the entire microgrid to supply loads. Because the electric power generated by all DGSs may be in different forms as DC and AC power, it should be converted into AC power with power inverters before connecting to the grid. Due to the requirement of system stability, all DGSs outputs have to be synchronized to the same magnitude and frequency. Therefore, load sharing has been adopted to operate and synchronize the set of parallel DGS power inverters.

So far, the research in literature on load 


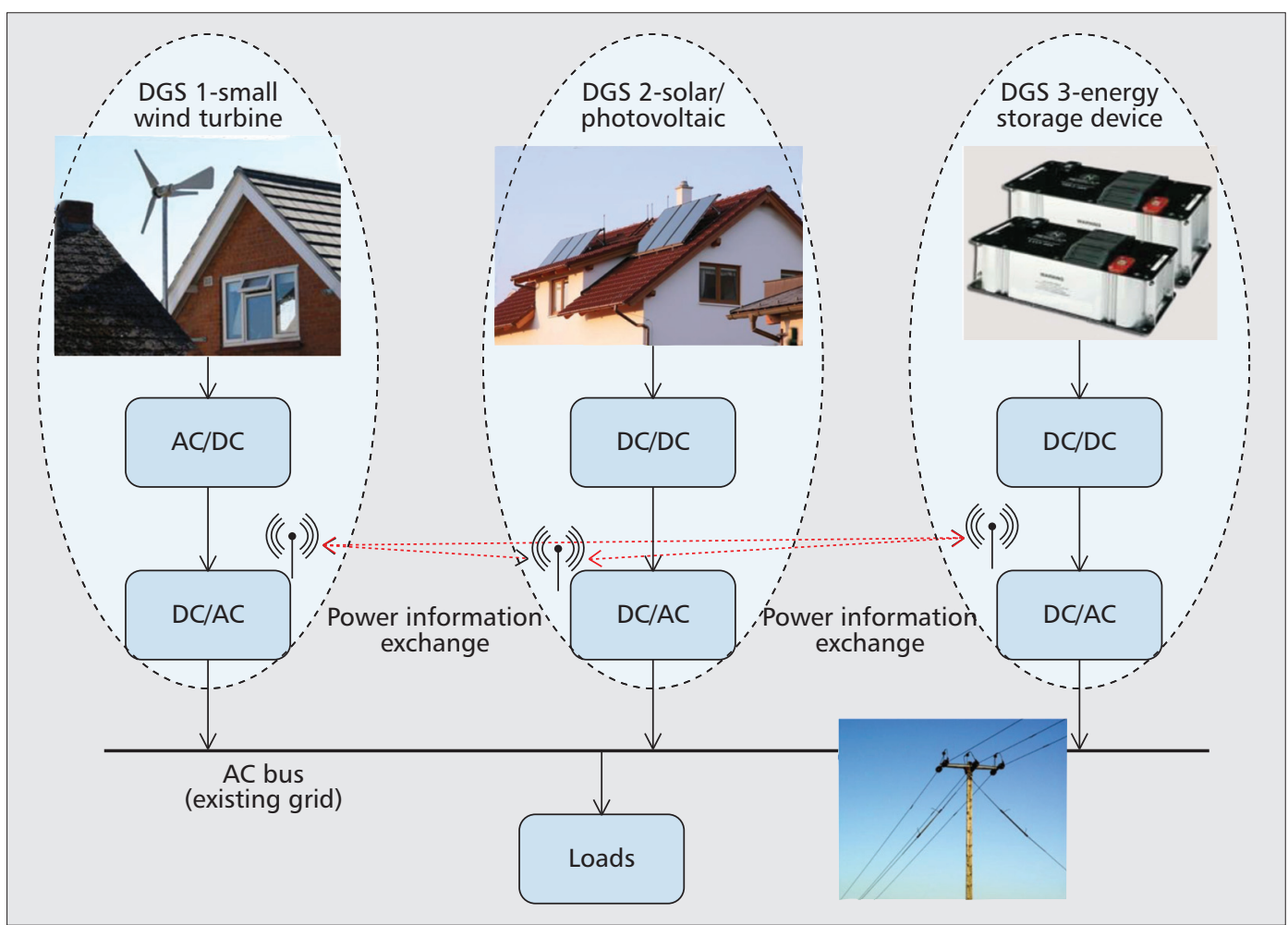

Compared with the

power converters

computation delay,

the wireless

communication delay

is much longer,

usually measured in

milliseconds.

Therefore it has

more significant

impact on the

performance of

the system.

Figure 1. A typical application scenario of load sharing in smart microgrid, where a wireless community mesh network is adopted for system information exchange among distributed generation systems (DGSs).

sharing has only been focused on better power control, and most of them assumed that wireless communication delay among different distributed generation sites is negligible or at a fixed value $[2,3]$. However, with the fast development of smart grids, the power control information usually shares the same wireless communication network along with other system information, meaning that the power control information may be delayed or even lost during the transmission. This should be taken into account, when designing a load sharing control system, for adequate outputs of parallel power inverters at different sites. Actually, all communication networks, especially wireless networks, proposed for smart grid communications, such as cellar, WiFi/WiMax, Internet, and power line communications, have time-varying communication delay. Based on this observation, it is crucial to study the parallel power inverters control in smart microgrids by taking into account the effect of the time-varying communication delay.

In this work, we will focus on the performance evaluation of load sharing among distributed generation systems through smart microgrids with considerations of the time-varying wireless communication delay. In this scenario, a smart microgrid is a wireless community network as shown in Fig. 1 where different DGSs provide electric power to the load and use the wireless community mesh network as the underlying wireless communication network. In this work, we adopt a wireless communication delay model to study the load sharing control of parallel inverters. To guarantee the operation stability of parallel inverters in distributed generation systems, we also propose a new controller design based on the Smith predictor to overcome the adverse effect of the time-varying wireless communication delay.

The rest of this article is organized as follows. We first briefly introduce the load sharing control in DGS, the time-varying wireless communication delay model, and the impact of wireless communication delay on power inverter control. We then discuss the proposed controller for load sharing with considerations of the time-varying wireless communication delay. Both theoretical and simulation results are provided to show the effectiveness and responsiveness of the proposed controller. Finally we conclude the article.

\section{PROBLEM DESCRIPTION}

In DGS, energy is generated by different distributed generation system units, such as wind turbines, solar/photovoltaic, energy storage devices or other sources, and is shared by the load through a microgrid as shown in Fig. 1. There exist several types of delay in the system shown in Fig. 1, including wireless communication delay, power converters computation delay, and electricity transmission delay. The electricity transmission delay is negligible in consideration of the high speed of electricity transmission. For the power converters computation delay, the authors in $[2,4]$ declared that this type of delay is less than half one PWM cycle and one PWM cycle is 185 us. Compared with the power converters computation delay, the wireless communication delay is much longer, usually measured in milliseconds. Therefore it has more significant impact on the performance of the system. As the 
microgrid only transmits AC power, DC/AC inverters are needed to convert $\mathrm{DC}$ power to $\mathrm{AC}$ power. According to [2, 4], the operation of parallel DC/AC inverters requires the load sharing control, in which different inverters at different locations have to exchange their output voltage/current information and then regulate their output voltages at the same magnitude and frequency.

In this section, the load sharing control of the parallel inverters will be first formulated and then the time-varying network delay model will be integrated into the load sharing control model. The impact of delay on control will be studied and analyzed.

\section{LOAD SHARING CONTROL OF PARALLEL DGS INVERTERS}

In order to ensure good load sharing of the real power and reactive power among different units, the average power control method has been widely adopted [2, 3]. Without losing generality, in this work, we adopt an application scenario, where two three-phase PWM inverters operating in parallel communicate with each other through a wireless community mesh network, as shown in Fig. 2a. In load sharing control, the power information is feedback from both local and remote inverters to generate the phase angle and magnitude of reference voltage for the optimal control. Specifically, the average value of active power information of local and remote power inverters combined with a constant is used to

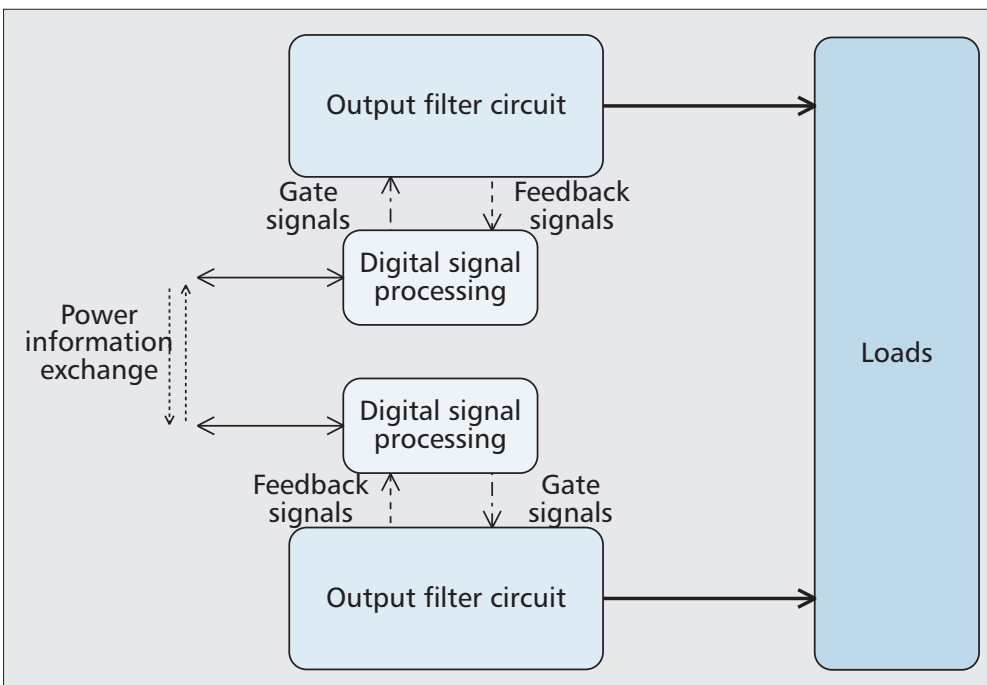

(a)

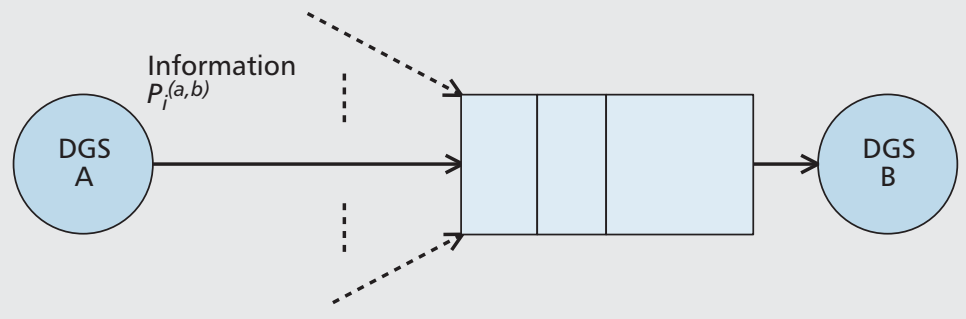

(b)

Figure 2. a) parallel inverters control with power information exchange; $b$ ) signal transmission delay model. generate the phase of the reference voltage, and the average value of reactive power information of local and remote power inverters with a constant is used to generate the magnitude of the reference voltage. According to the control timing diagram described in [4], the optimal load sharing control requires the power information from both local and remote inverters, which means that the power information should be sampled and delivered on time to generate the reference voltage and then the PWM signals. As the power information of remote DGS units is transferred over the wireless communication network, there are time delay incurred by information packet or possible information missing, which means that the reference voltage is generated in error.

\section{Signal TRansmission Delay}

Since the parallel inverters need to share the power information among them, the timely delivery of power information of different inverters is important. Without losing generality, in this work we consider a delay model of wireless networks to characterize the signal transmission delay over a lossy wireless communication channel.

In the wireless network environment, as shown in Fig. 2b, the transmission of one packet over one wireless link $(a, b)$ of the communications network can be modeled as follows: the packet arrives at node a (DGS a unit), waits in the queue of node a, and is transmitted/served over link $(a, b)$. In consideration of the requirement of power inverters on the delay performance of power information, we define that each packet is associated with a delay deadline $T^{\text {budget }}$. In other words, if the packet arrives after the delay deadline $T^{\text {budget }}$, it will be considered of no use for the load sharing control. If the packet gets lost during transmission, it will be retransmitted until it is either successfully received or discarded because its delay deadline $T^{\text {budget }}$ was exceeded. During the above period, the total packet loss probability $P_{i}^{(a, b)}$ incurred by packet i mainly consists of two parts:

- The probability of packet drop $p_{i}^{a}$ due to delay deadline expiration when queuing at node a

- The probability of packet loss $P_{i}^{(a, b)}$ over link $(a, b)$ which is mainly determined by packet error probability $P_{i}^{(a, b) \_ \text {err }}$ due to signal fading over link $(a, b)$

In wireless networks, $P_{i}^{(a, b) \_r r}$ depends on specific channel conditions, indicated by Signal-toInterference-Noise-Ratio (SINR), and the adopted modulation and channel scheme. To deal with $p_{i}^{(a, b)}$ over link $(a, b)$, retransmission mechanisms such as the selective repeat algorithm [5] are commonly used at node $a$ within the packet delay deadline. Therefore, the delay incurred by packet $i$ is mainly caused by the retransmissions over link $(a, b)$.

To derive the incurred delay of packet $i$ over link $(a, b)$, we need to calculate the allowable maximum number of retransmissions of packet $i$ within its delay deadline $T^{\text {budget }}$. Let $R^{(a, b)}$ be the transmission rate of link $(a, b)$, and $L$ the length of packet $i$. Then, as described in [6], the maximum retransmission limit $\tau_{i}^{(a, b)}$ can be derived as a function of $\left\{R^{(a, b)}, L, T^{\text {budget }}\right\}$. 
We consider the service time $X_{i}^{(a, b)}$ for packet $\pi_{l}^{i}$ over link $(a, b)$ as a geometric distribution. So the first and second moments of the service time

$$
E\left[X_{i}^{(a, b)}\right] \text { and } E\left[\left(X_{i}^{(a, b)}\right)^{2}\right]
$$

can be calculated as in [6]. By queuing analysis [6], the average waiting time for packet $\pi_{l}^{j}$ at node a can be shown as

$$
E\left[W_{i}^{(a, b)}\right]=\frac{\lambda^{a} E\left[\left(X_{i}^{(a, b)}\right)^{2}\right]}{2\left(1-\lambda^{a} E\left[X_{i}^{(a, b)}\right]\right)},
$$

where $\lambda^{a}$ is the average packet arrival rate at node a. The values of $\lambda^{a}$ can be locally obtained at node a by counting and averaging the total number of incoming packets over a certain period. The final total delay $T_{\text {delay }}$, which can be expressed as the combination of the service time and average waiting time is the time that the power information transmission between different DGS units $a$ and $b$ takes.

Different from previous literatures [2, 3] where the delay time is assumed to be a fixed value or negligible, our model can effectively characterize the actual time-varying delay under different wireless channel conditions.

\section{IMPACT OF WIRELESS COMMUNICATION DELAY ON LOAD Sharing CONTROL}

As mentioned earlier, the wireless communication delay will cause the parallel inverters to use outdated power information to generate the reference voltage for load sharing control. As a result, the reference voltage is expressed as the relationship of active/reactive power of local and remote inverters. Notice that the power information of remote inverter is missed.

From the previous analysis on load sharing control of parallel DGS inverters, we can observe that the generated reference voltage will change in both magnitude and phase due to the network transmission delay, indicating that the outdated power information disables the controller from outputting the optimal power for load sharing. The controller will work in the suboptimal condition until the remote power information is timely delivered to the local DGS unit. During this period, the suboptimal output of the local DGS unit may be harmful. Thus, a new controller needs to be designed to overcome the delay of remote power information and to keep the load sharing system stable.

\section{The Proposed Controller Design}

So far, we have studied the impact of wireless communication delay among parallel power inverters on load sharing control. As indicated in the previous section, the delay may result in suboptimal output power information for load sharing. In this work, we design a stable controller for parallel power inverters to overcome the impact of the wireless communication delay, as

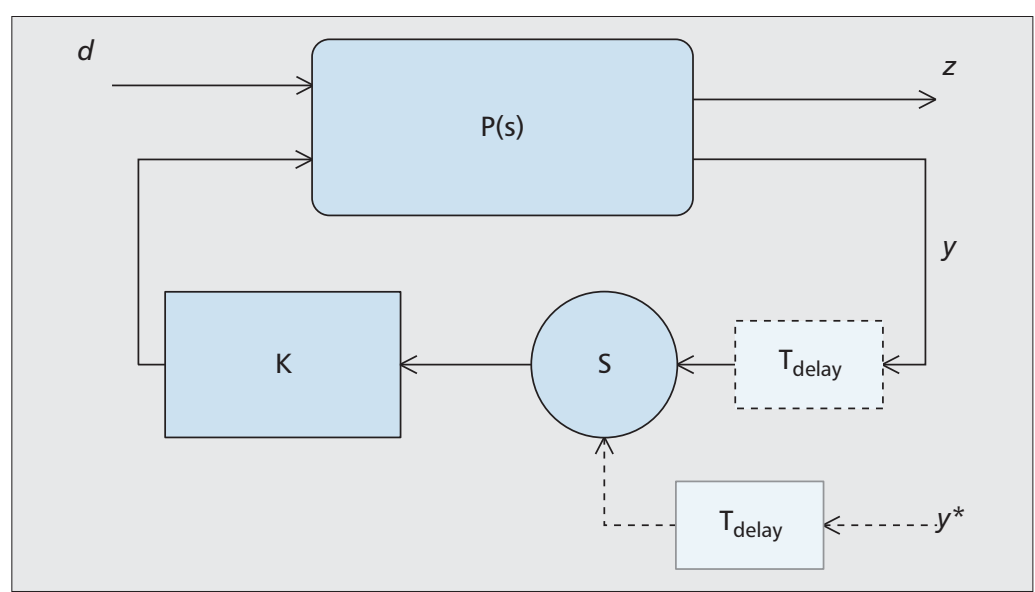

Figure 3. The proposed load sharing controller, and the dotted pink one is the introduced delay block compared with the existing controller.

shown in Fig. 3. The design idea of this controller is to make the feedback signal from both remote and local inverters arrive at the controller synchronously, which is realized by delaying the arrival time of the local feedback information.

Because the controller designed is to deal with the delay of feedback signal, we formulate the problem from transfer matrix of the control. The general control setup for operation of parallel inverters having signal transmission delay is shown in Fig. 3, where $P(s)$ stands for the 2 dimension control matrix made up of $P_{11}, P_{12}$, $P_{21}$ and $P_{22}$.

The transfer matrix of the proposed controller from $d$ to $z$ is $T_{z d}(s)=P_{11}+P_{12} \cdot K \cdot T_{d e-}$ lay $\cdot\left(\mathrm{I}-P_{22} \cdot K \cdot T_{\text {delay }}\right)^{-1} \cdot P_{21}$, where $T_{\text {delay }}$ is the signal transmission delay. The transfer matrix $T_{z d}(s)$ indicates that the response through $P_{11}$ has no delay, as shown in Fig. 4a, while the wireless communication delay has impact on the path $P_{21}-s-K-P_{12}$ through $P_{22}$. An equivalent structure of the proposed structure is shown in Fig. 4a. To obtain a stable system output, a possible solution is to maintain uniform delay in all paths. In this work, a random variable obeying the same distribution of the wireless communication delay is introduced to the local control loop, as shown in Fig. 3. The random distribution used to generate the random delay can be derived from the wireless communication delay model presented in previous section. With the introduced delay, the local feedback information will take longer time to arrive the control module. Using this approach, both local and remote feedback signals will statistically arrive at the controller simultaneously. The generation of the random delay based on the wireless communication delay model is realized by the power inverter control module.

Here, $y^{\prime}$ is the average load feedback, standing for both local power information $\mathrm{y}$ and remote inverter power information $y^{*}$. A unified smith predictor approach is adopted in this work to formulate the control design problem, and the overall control setup is based on the analysis we have illustrated. To formulate this problem with unified smith predictor approach, first we need to decompose the plant into critical and noncriti- 


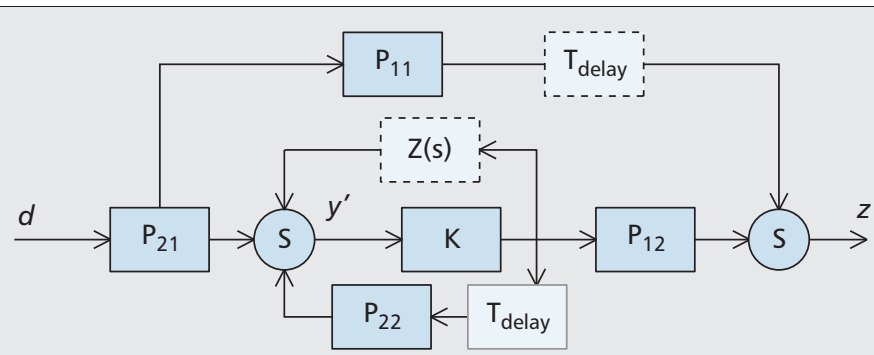

(a)

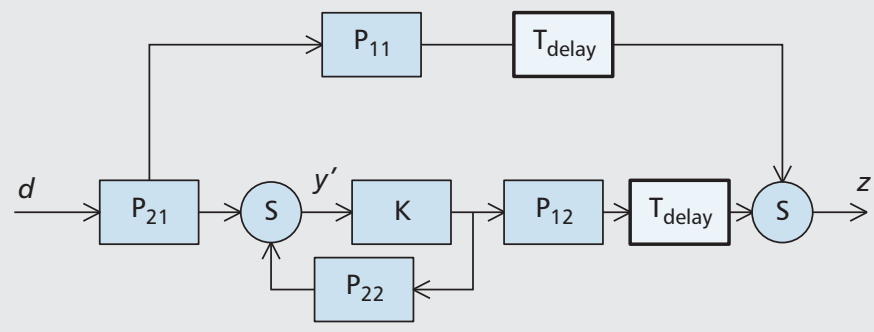

(b)

Figure 4. a) Equivalent representation of the proposed load sharing controller with the dotted blocks designed to obtain uniform delay time; $b$ ) equivalent representation of the proposed controller with uniform delay time.

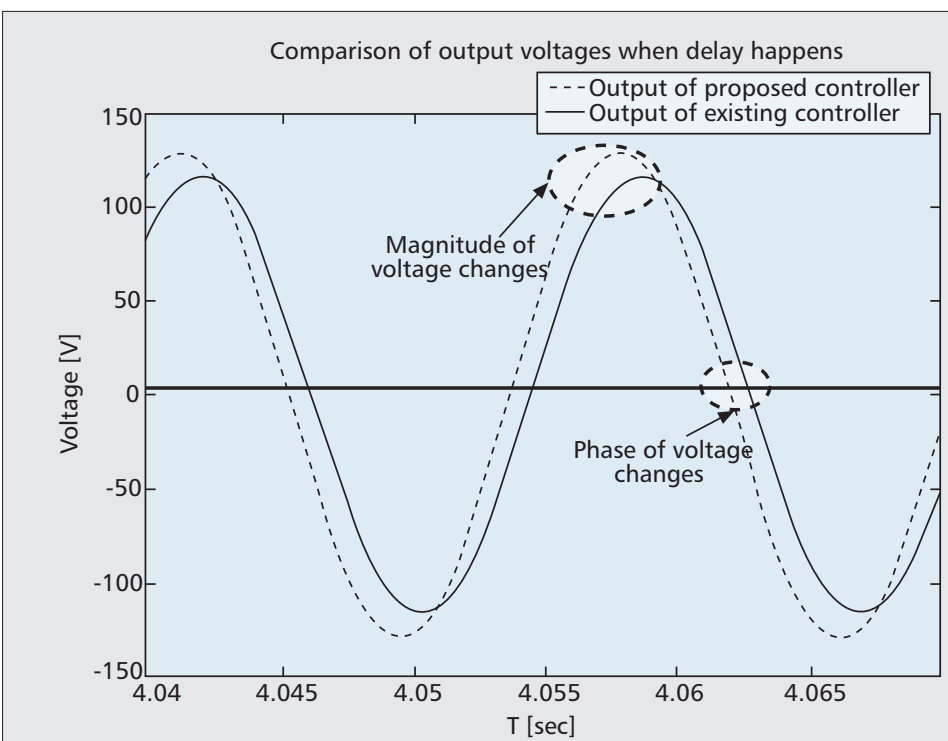

(a)

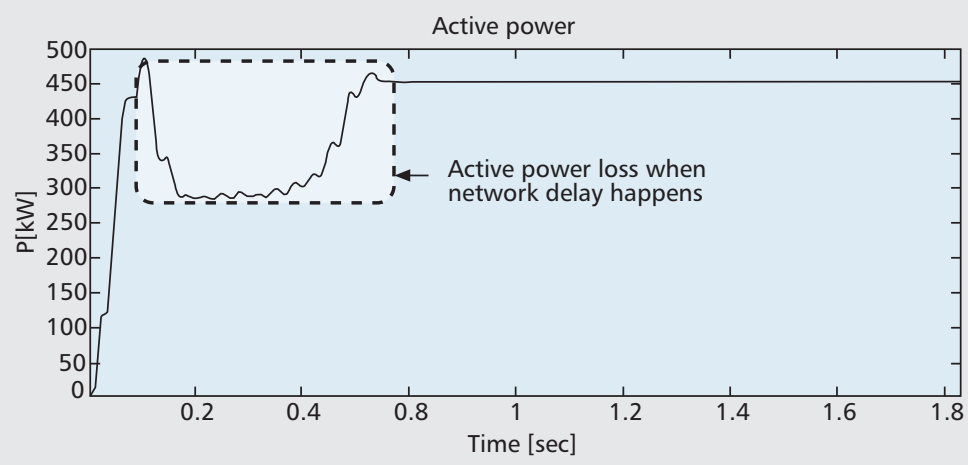

(b)

Figure 5. a) Comparison of the proposed controller vs an existing controller; $b$ ) The lost of active power under the network delay in DGS. cal parts. This can be done by applying a suitable linear coordinate transformation on the statespace model of the plant.

The formulated problem can be solved by introducing a unified Smith Predictor [7] to shift the position of delay in path $P_{21}-s-K-$ $\mathrm{P}_{12}$ and a delay block in path $P_{11}$ to maintain uniform delay in both paths. The unified Smith Predictor can be written as $Z(s)=P_{22}(\mathrm{~s})-$ $P_{22}(s) T_{\text {delay }}$ as shown by the dotted box in Fig. $4 \mathrm{a}$. Then, a generalized control plant is formed, in which both control paths have the same statistical delay time, as shown in Fig. $4 \mathrm{~b}$. The following steps to get the final formulation of the control block are described in [8] in detail.

\section{SimULATION ResUlt}

In this work, extensive simulations have been carried out to study the impact of the wireless communication delay and the performance of the proposed controller. Simulations are performed using Matlab/Simulink with power system block-set. This model is composed of two DGS units, two loads, and the wireless communication delay. The nominal values of the circuit components for simulations are configured as follows. The rated output power of each DGS system is $600 \mathrm{k}$ VA. The AC load voltage is $480 \mathrm{~V}$ and the $\mathrm{V}_{\mathrm{DC}}$ is $2200 \mathrm{~V}$ ac. The PWM frequency is 5.4 $\mathrm{kHz}$ corresponding to a sampling period $T_{s}=$ $185 \mu \mathrm{s}$.

Figure 5a shows the output voltage of the power inverter with and without the remote inverter's power information, where the dotted line indicates the output inverter voltage without signal transmission delay. From this figure we can observe that both phase and magnitude of the output voltage are shifted from the optimal values with considerations of the wireless communication delay. Furthermore, in Fig. 5b the active power of the DGS unit drops significantly due to the delay, which may lead the system to be unstable for a certain period in DGS and may cause serious damages to the energy consumption devices. This figure illustrates two possible adverse impacts of the wireless communication delay: the suboptimality and instability of the DGS system output. The simulation results illustrate the impact of this time-varying delay on the output of the distributed generation system. According to the analysis, when the wireless communication delay happens, the reference voltage of the power inverter in the DGS changes, and as a result the output of the inverter will change with wrong magnitude and frequency values.

Figure 6 illustrates the active power output by using the proposed controller. Compared with the existing controller output, we can observe that although the active power output may have ripples when the wireless communication delay is incurred, the active power output does not drop much and can be recovered much faster. The lost output power as shown in Fig. 5b is compensated by the proposed controller. Furthermore, compared with the existing system, the required active power can be recovered more quickly, meaning that the DGS 
using the proposed controller is much more stable with considerations of the wireless communication delay.

The deteriorated performance of the distributed generation system reveals the fact that the effect of the time-varying wireless communication delay is essential to load sharing among distributed generation systems. If it is not taken into account at the design stage the DGS might not perform as expected. Therefore, it is necessary to consider the wireless communication delay in the control design formulation. The results demonstrate a potential application of the uniform smith predictor approach for power inverters control in distributed generation systems involving the time-varying wireless communication delay.

\section{CONCLUSIONS}

Existing work in the area of load sharing among distributed generation systems (DGS) in wireless enabled smart microgrids has largely ignored the effect of the wireless communication delay. Study has shown that the time-varying wireless communication delay may have a significant impact on the performance of power inverters. In this article, we have studied the impact of the time-varying wireless communication delay on the load sharing control. Based on the impact analysis, we also have proposed a new controller that can properly control the load sharing among distributed generation systems through wireless-enabled smart microgrids with considerations of the time-varying wireless communication delay. The proposed controller is designed by maintaining a uniform delay in all transfer paths through a unified Smith predictor approach. The simulation results have verified the effectiveness and responsiveness of the proposed control strategy in overcoming the adverse impact of the time-varying wireless communication delay on the performance of distributed power inverters.

\section{ACKNOWLEDGEMENTS}

This work was partially supported by NSF under Grant CCF no. 0830493 and CNS no. 1145596 and NSFC under Grant no. 60972083.

\section{REFERENCES}

[1] H. Farhangi, "The Path of the Smart Grid," IEEE Power and Energy Mag., vol. 8, Jan. 2010, pp. 18-28.

[2] M. Marwali, J. Jung, and A. Keyhani, "Control of Distributed Generation Systems-Part II: Load Sharing Control," IEEE Trans. Power Electronics, vol. 19, Nov. 2004, pp. 1551-61.

[3] Y. Byun et al., "Parallel Operation of Three-Phase UPS Inverters by Wireless Load Sharing Control," Proc. Telecommunications Energy Conf., Phoenix, AZ, Sept. 2000, pp. 526-32.

[4] M. Marwali, J. Jung and A. Keyhani, "Control of Distributed Generation Systems-Part I: Load Sharing Control," IEEE Trans. Power Electronics, vol. 19, Nov. 2004, pp. 1541-50.

[5] A. Tanenbaum, Computer Networks, Upper Saddle River, NJ: Prentice-Hall, 2003.

[6] D. Wu et al., "Application-Centric Routing for Video Streaming over Multi-hop Wireless Networks," IEEE Trans. Circuits and Systems for Video Tech., vol. 20, no. 12, Dec. 2010, pp. 1721-34.

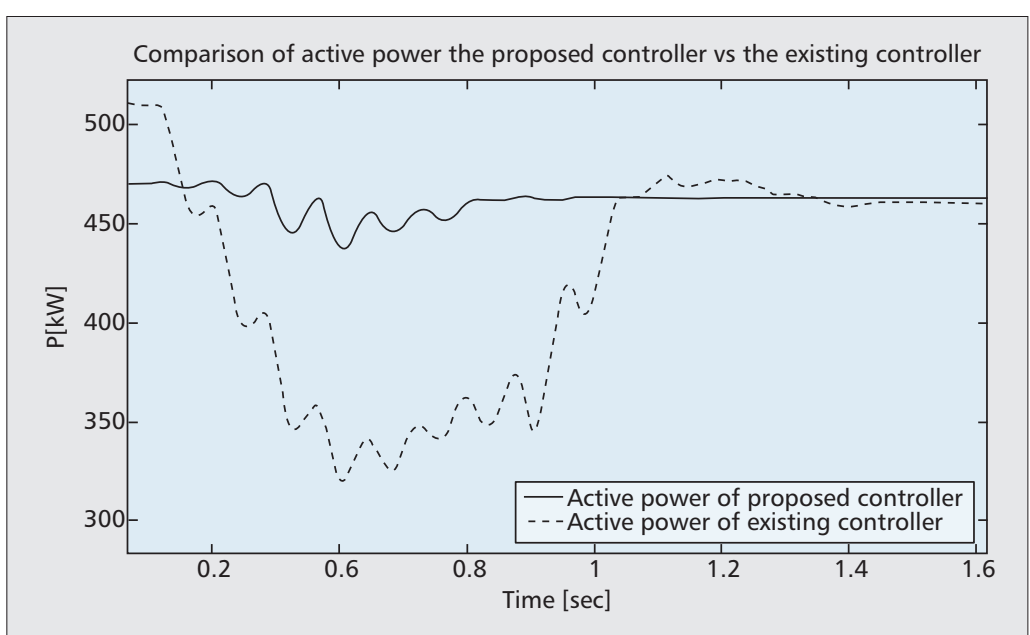

Figure 6. Comparison of the active power of proposed controller vs an existing controller.

[7] B. Chaudhuri, R. Majumder, and B. Pal, "Wide-Area Measurement-based Stabilizing Control of Power System Considering Signal Transmission Delay," IEEE Trans. Power Systems, vol. 19, Nov. 2004, pp. 1971-79.

[8] Q. Zhong and G. Weiss, "A Unified Smith Predictor based on the Spectral Decomposition of the Plant," Int'l. J. Control, vol. 77, Oct. 2004, pp. 1362-71.

\section{BIOGRAPHIES}

SONG CI [S'98, M'02, SM'06] (sci@engr.unl.edu) received his B.S. from Shandong University of Technology (now Shandong University), Jinan, China, in 1992, M.S. from Chinese Academy of Sciences, Beijing, China, in 1998, and Ph.D. from the University of Nebraska-Lincoln in 2002, all in Electrical Engineering. Currently, he is an Associate Professor of Computer and Electronics Engineering at the University of Nebraska-Lincoln. His research interests include: dynamic complex system modeling and optimization, green computing and power management, dynamically reconfigurable embedded system, content-aware quality-driven cross-layer optimized multimedia over wireless, cognitive network management and service-oriented architecture, and cyber-enable e-healthcare.

JUNJIE QIAN (junjie.qian@huskers.unl.edu) received his B.S degree in Electronics Science and Technology and M.E. in Micro-electronics from Xi'an Jiaotong University, China, in 2005 and 2010, respectively. He is currently working toward the Ph.D. degree in the Department of Computer Science and Engineering, the University of Nebraska-Lincoln. His research interests include Computer Architecture, Runtime Systems and Virtual Machine, Analog/Digital Circuit Design.

DALEI Wu [S'05, M'10] (daleiwu@mit.edu) received his B.S and M.E. degrees in electrical engineering from Shandong University, Jinan, China, in 2001 and 2004, respectively, and Ph.D. degree in Computer Engineering from the University of Nebraska-Lincoln in 2010. Currently, he is a Postdoctoral Research Associate with the Mechatronics Research Lab, Massachusetts Institute of Technology. His research interests include cyber-physical systems, wireless sensor networks, and multimedia computing and networking.

Alı Keyhani [S'72, M'76, SM'89,F'98] (keyhani@ece. osu.edu) received the B.E., M.S.E.E., and Ph.D. degrees from Purdue University, West Lafayette, IN, in 1967, 1973, and 1976, respectively. Currently, he is a Professor of electrical engineering at The Ohio State University (OSU), Columbus. He is also the Director of the OSU Electromechanical and Green Energy Systems Laboratory. His current research interests include control of distributed energy systems, power electronics, multilevel converters, power systems control, alternative energy systems, fuel cells, photovoltaic cells, microturbines, distributed generation systems, and parameter estimation and control of electromechanical systems. 